Research Article

\title{
PTS-FNN-Based Health Prediction Method for Flexible Photoelectric Film Material Processing Equipment
}

\author{
Yaohua Deng $\mathbb{D}^{1}{ }^{1}$ Kexing Yao $\mathbb{D}^{1},{ }^{1}$ Tuo Jin $\left(\mathbb{D},{ }^{2}\right.$ Zhaoxi Feng $\mathbb{D}^{1},{ }^{1}$ and Xiali Liu ${ }^{1}{ }^{1}$ \\ ${ }^{1}$ School of Electro-mechanical Engineering, Guangdong University of Technology, Guangzhou, Guangdong, China \\ ${ }^{2}$ Zhejiang Tobacco Company Wenzhou Company, Wenzhou, Zhejiang, China
}

Correspondence should be addressed to Kexing Yao; 2111701138@mail2.gdut.edu.cn

Received 9 December 2019; Accepted 16 March 2020; Published 23 April 2020

Academic Editor: Stylianos Georgantzinos

Copyright ( $\odot 2020$ Yaohua Deng et al. This is an open access article distributed under the Creative Commons Attribution License, which permits unrestricted use, distribution, and reproduction in any medium, provided the original work is properly cited.

Roll-to-Roll (R2R) processing is a common processing method for flexible photoelectric film materials. Due to the physical properties of the materials, the change in the performance of the $\mathrm{R} 2 \mathrm{R}$ processing equipment can easily cause deformation of the flexible film material, it is particularly important to predict the performance degradation of the processing equipment. Based on the accuracy and real-time requirements of performance degradation prediction, a PTS-FNN model for performance degradation prediction was proposed in this paper, which combines the Possibilistic C-Means (PCM) fuzzy clustering and Takagi-Sugeno Fuzzy Neural Network (TS-FNN). We also studied the PCM classification algorithm of input data of PTS-FNN model, the predecessor network of TS-FNN prediction model and the construction method of postcomponent network. Finally, the implementation process of PCM classification algorithm and TS-FNN prediction model were given. The R2R processing equipment health prediction experiment system was built and the PTS-FNN model experiment was carried out. The experimental results showed that the training time of PTS-FNN model was $50.37 \%$ less than the standard TS-FNN prediction model. The prediction accuracy increased by $5.48 \%$, and the PTS-FNN had no error in the judgment of state 1 and state 4 .

\section{Introduction}

Currently, Roll-to-Roll (R2R) processing technique is the most widespread processing method for a series of flexible thin film materials internationally, for it can maintain the enhancement its productivity while automatizing the mechanical equipment to the greatest extent. R2R processing is a common processing method for flexible photoelectric thin film materials, and the performance deterioration of the $\mathrm{R} 2 \mathrm{R}$ processing device is the primal problem the mass manufacturing of flexible photoelectric thin film materials faces. In recent years, the technology of equipment health prognosis has become a research hotspot $[1,2]$. In 2017, Lee et al. [3] proposed a prognosis algorithm that boosts material removal rate (MRR) based on integrated models and data-driven approach. The method proposed combines the influences of physical mechanism and nearest neighbors, extracts the relating characteristics.
These characteristics are inputted to build multiple regression models which will be integrated so as to obtain the final prognosis. Rapid development and extensive applications in deep learning have been achieved in the last few years. In the field of industry, the researcher applies deep learning to the analysis of industrial data. University of Michigan's Ni Xia [4] developed an operating load based real-time rolling Grey forecasting technique, so as to provide efficient accurate machine health prognosis, as well as analyzed the influencing factors. Deng et al. [5] (2018) combined the quality control chart and SoV, proposing a fault diagnosis approach of the flexible material $\mathrm{R} 2 \mathrm{R}$ manufacturing system. Based on the relativity between the source of fault and product quality during manufacturing as well as statistical distribution pattern of the feature vector of processing quality and the source of fault, this approach utilized SoV model under controlled or uncontrolled state and mathematical model of probability 
distribution to deduce the statistical measurement of quality characteristic variables. The consequent quality control chart can pinpoint the fault location in the system quickly and easily. Zhao and Ouyang [6] (2016) applied Multi-Agent Genetic Algorithms (MAGA) to the prognosis of the state of avionic devices, which overcomes the flaw of the Baum-Welch algorithm easily falling into locally optimal solutions, greatly enhancing the prognosis accuracy, speed, and stability.

In application, the health prognosis model for $\mathrm{R} 2 \mathrm{R}$ processing device has advanced instantaneity requirement; therefore, it is particularly vital to study how to enhance the instantaneity of the prognosis model. Because the Fuzzy C-Means (FCM) clustering algorithm is based on fuzzy theory to describe the uncertainty of sample generics, the fuzzy membership value of each classification point is obtained by optimizing the objective function. Although FCM is widely used in clustering algorithms, it has the problem of being sensitive to noise data. The PCM clustering algorithm can make up for the shortcomings of the above-mentioned FCM algorithm by relaxing the constraint of membership degree without normalizing the data. Because the neural network modeling has the characteristics to deal with the nonlinear approximation problem well, the PCM clustering method can well divide the data with irregular boundaries. For that reasons, the following content will be binding the PCM clustering analyzing method and the Takagi-Sugeno Fuzzy Neural Network (TS-FNN) to discuss the health state prognosis modeling of the R2R processing device.

\section{Neural Network Model for Health Prediction of Roll-To-Roll Processing Equipment}

The standard TS-FNN [7] was first proposed by Japanese scholars Takagi and Sugeno; it is a specific method for identifying fuzzy models of nonlinear dynamic systems. TS-FNN can be divided into antecedent networks and consequent network according to different structural functions (Figure 1). The antecedent network is used to match the fuzzy rules, that is, to calculate the adaptability of each fuzzy rule. The antecedent network is composed of four layers of neural networks: the first layer is the input layer, the second layer is the membership layer (Gaussian function), the third layer is to calculate the adaptability $\left(a_{j}=\min \left\{u_{11}, \ldots u_{1 j}, \ldots u_{n j}\right\}, a_{j}\right.$ is the $j^{\text {th }}$ fuzzy rule adaptability, $n$ represents the number of inputs), the fourth layer is to perform normalized processing, the adaptability is normalized $\left(\overline{a_{j}}=\left(a_{j} / \sum_{b=1}^{m} a_{b}\right), m\right.$ represents the number of nodes in the fourth layer of antecedent network), and $\left\{\overline{a_{1}}, \overline{a_{2}}, \ldots \overline{a_{m}}\right\}$ is the output to the antecedent network. The consequent network is to generate fuzzy rules, it consists of three layers of networks. The first layer is the input layer, and the second layer is the rule computing layer $\left(y_{g j}=\sum_{i=0}^{n} p_{j i}^{r} x_{i}, j=1,2, \ldots m ; g=1,2, \ldots r ; p_{j i}^{r}\right.$ is the linear parameter of the consequent network). The third layer is the system output layer. The system output $\left\{y_{1}, y_{2}, \ldots y_{r}\right\}$ is obtained by adding the weights of $\left\{\overline{a_{1}}, \overline{a_{2}}, \ldots \overline{a_{m}}\right\}$ as the connection weights, where $y_{r}=\sum_{j=1}^{m} \frac{m}{a_{j}} y_{r j}, y_{r} \in Y, y_{r j}$ is the output of the $j^{\text {-th }}$ fuzzy rule.

The standard T-S fuzzy neural network model requires to linearize the input data, which will make the input space and fuzzy rules difficult to optimize and extract, and this will lead to more problems, for instance, the system structure should be planned in advance. Therefore, when dealing with the multi-input nonlinear space, the PCM fuzzy clustering is used as the antecedent model extraction method, to realize the expected purpose of improving the training efficiency and recognition effect of the model. The PCM algorithm can adjust the clustering center, radius, and number of clusters of the input space; rationally divide the ambiguity of the input data; and determine the membership function of the data points and the rule adaptability; therefore, this PCM algorithm achieves basic elimination of the low quality samples to participate in the antecedent networks calculation, and model training speed and accuracy can be greatly improved. According to the above analysis and reference [8], the performance regression evaluation model PTS-FNN of R2R processing equipment based on PCM and TS-FNN shown in Figure 2 is proposed. The model combines the advantages of the PCM method, the T-S fuzzy inference model, and the fuzzy neural network modeling method. The PTS-FNN model includes an antecedent network and a consequent network. The antecedent network matches the standard T-S fuzzy rules, and the antecedent network concludes the fuzzy rules. After the weighted calculation of the antecedent and consequent network, the PCA parameter of each station roller axis performance $X=\left\{x_{1}, x_{2}, \ldots x_{n}\right\}$ and the nonlinear modeling of equipment health status $S=\left\{s_{1}\right.$, $\left.s_{2}, \ldots s_{r}\right\}$ are established [9].

\section{PCM Classification Method for Input Data of Health State Prediction Model of Roll-To-Roll Processing Equipment}

Instead of normalizing the input data, Possibilistic C-Means Clustering Algorithm relaxes the constraints of the membership function during the division of data, which is an advantage of Possibilistic C-Means Clustering Algorithm, thus enhancing the sensitivity to noisy data $[10,11]$.

Here are the assumptions that $x_{h}$ of the sample set $X=$ $\left\{x_{1}, x_{2}, x_{3}, \ldots, x_{h}, \ldots x_{n}\right\}$ possesses $p$ amount of characteristics $x_{h}=\left\{x_{h 1}, x_{h 2}, x_{h 3}, \ldots, x_{h p}\right\}$, the sample set $X$ possesses $c$ amount of clustering center $V=\left\{v_{1}, v_{2}, v_{3}, \ldots, v_{c}\right\}$, and using $d_{i k}$ to represent the Euclidean distance of sample $x_{k}$ and clustering center $v_{i}$, gives

$$
d_{i k}=\left\|x_{k}-v_{\mathbf{i}}\right\|=\sqrt{\sum_{j=1}^{p}\left(x_{k j}-v_{i j}\right)^{2}} .
$$

In equation (1), $i=1,2, \cdots, c ; j=1,2, \cdots, p ; k=1,2, \cdots, n$. Then the target function of PCM: 


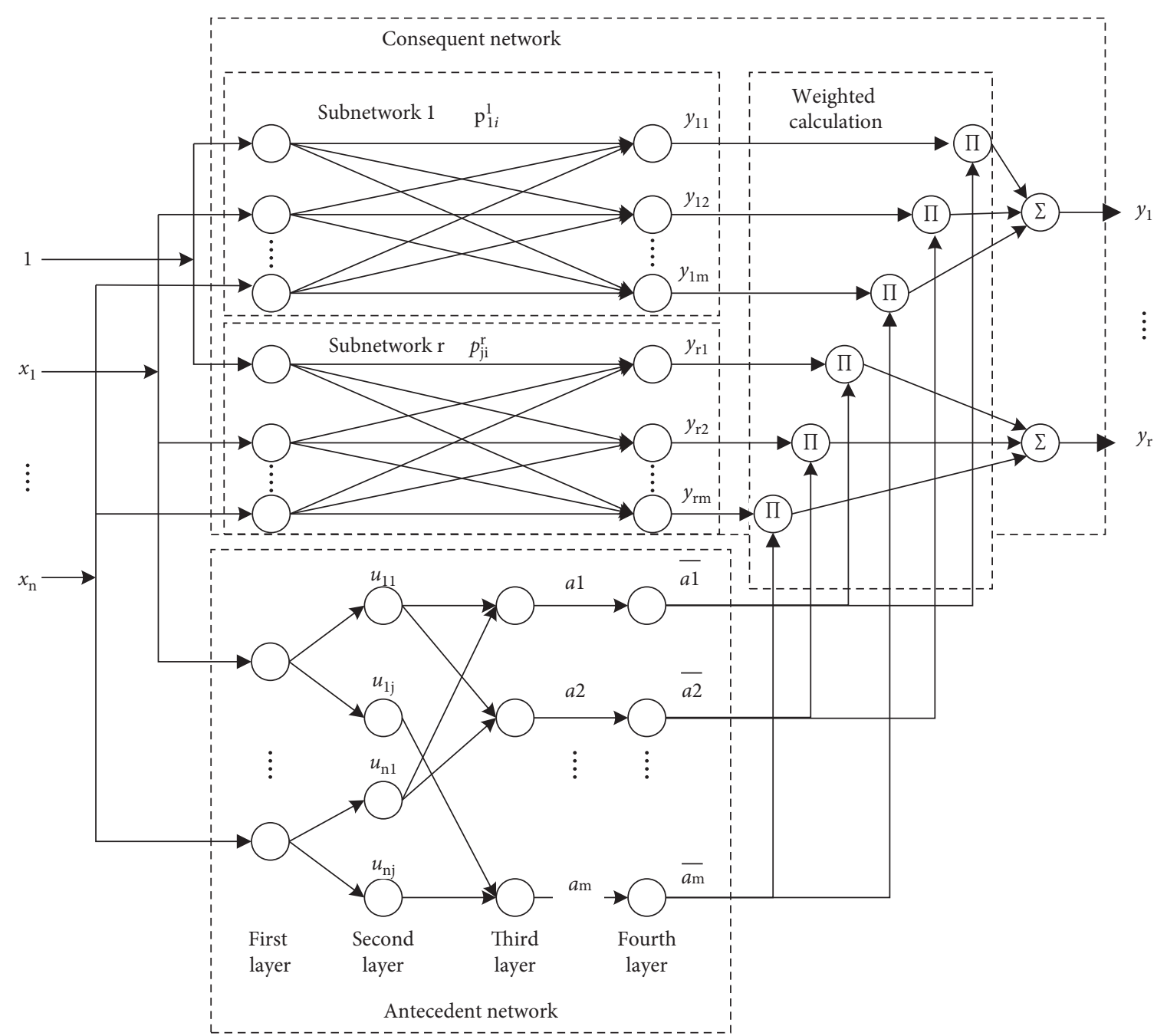

FIgURE 1: T-S fuzzy neural network model.

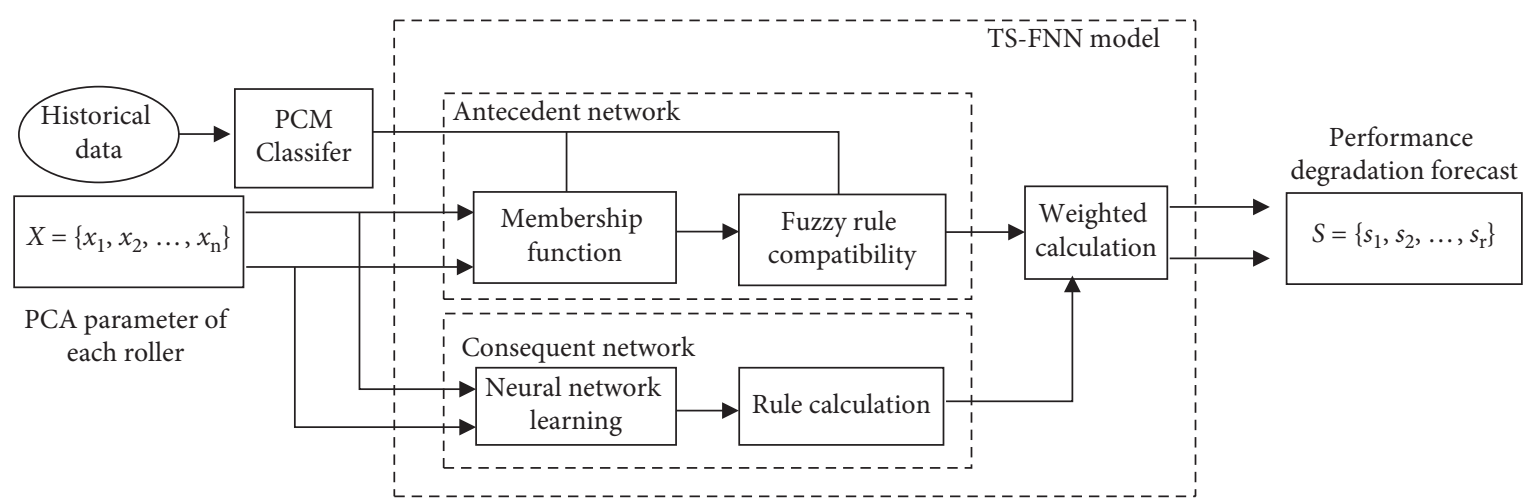

FIGURE 2: PTS-FNN-based R2R processing equipment health prediction model framework.

$\min J_{\mathrm{PCM}}(X, U, V)=\sum_{i=1}^{c} \sum_{k=1}^{n}\left(u_{i k}\right)^{\boldsymbol{\alpha}} d_{i k}^{2}+\sum_{i=1}^{c} \eta_{i} \sum_{k=1}^{n}\left(1-u_{i k}\right)^{\boldsymbol{\alpha}}$.

In equation (2) $U=\left[u_{i k}\right]_{c \times n}$ represents the possibility partition matrix; $\alpha$ represents the weighting factor of the clustering's ambiguity and $\alpha \in[1, \infty]$ (normally $1.5 \leq \alpha \leq 2.5) ; u_{i k}$ represents membership under category No. $i$ sample No. $\mathrm{k}\left(u_{i k} \in[0,1]\right) ; \eta_{i}$ represents the penalty parameter, its expression is shown as equation (3) [12].

$$
\eta_{i}=K \frac{\sum_{k=1}^{n}\left(u_{i k}\right)^{\alpha} d_{i k}^{2}}{\sum_{k=1}^{n}\left(u_{i k}\right)^{\alpha}}
$$


In order to avoid null solution in the target function, introducing the regularization term $f(U)$ of the possibility partition matrix (shown in equation (4)), provides a larger penalty value when $u_{i k} \longrightarrow 0^{+}$.

$$
f(U)=\sum_{i=1}^{c} \eta_{i} \sum_{k=1}^{n}\left(1-u_{i k}\right)^{\alpha} .
$$

As mentioned in the previous chapters and sections, for the sake of dividing the fuzzy level of the input data of the health prognosis artificial neural network model for R2R processing device precisely, the front end of the PTS-FNN model is classified by PCM first. Combining equations (1)-(4), the calculating formulas of the possibility partition matrix $U$ and the clustering center $V$ is shown as equations (5) and (6), respectively.

$$
\begin{aligned}
u_{i k} & =\left[1+\left(\frac{d_{i k}^{2}}{\eta_{i}}\right)^{(1 / \alpha-1)}\right]^{-1}, \\
v_{i} & =\frac{\sum_{k=1}^{n}\left(u_{i k}\right)^{\alpha} x_{k}}{\sum_{k=1}^{n}\left(u_{i k}\right)^{\alpha}} .
\end{aligned}
$$

Figure 3 provides the process of the PCM algorithm:

(1) Set clustering category to $c$, set the fuzzy weight to $\alpha$ (normally $\alpha=2$ ), set the iteration thresholding to $q$ (normally between 0.001 and 0.01 ), set the iteration count to $t$, initialize the possibility partition matrix $U^{(s)}$ and the clustering prototype $V^{(s)}(s=0)$

(2) Estimate $\eta_{i}$ base on $U^{(s)}$ and $V^{(s)}$ as well as equation (3)

(3) Update the division of the matrix $U^{(s+1)}$ and the clustering prototype $V^{(s+1)}$ in accordance with equations (5) and (6)

(4) Reestimate $\eta_{i}$, repeat (3)

(5) Determining threshold: according to the given threshold $q$, if $\left\|V^{(s+1)}-V^{(s)}\right\| \leq q$, stop the iteration; otherwise, $t=t+1$ and Jump to (2)

\section{Construction of TS-FNN Model for Health State Prediction of Roll-To-Roll Processing Equipment}

Figure 2 shows that the health status prognosis TS-FNN model for R2R processing device includes determining the structural parameter of the antecedent network and the consequent network. The following content focus on introducing the deduction of the antecedent network's the membership function and the rule compatibility, as well as the weight between each node of the consequent network can be obtained using the Error Back Propagation Algorithm [8].

Here are the assumptions that matrix $\mathrm{U}=\left[u_{i k}\right]$ is a fuzzy matrix after PCM data division, $G_{i}(1 \leq i \leq c)$ represents fuzzy category group of $c$ after division, $\beta$ represents clustering fuzzy degree weighted index and the category center $v_{i q}$ and

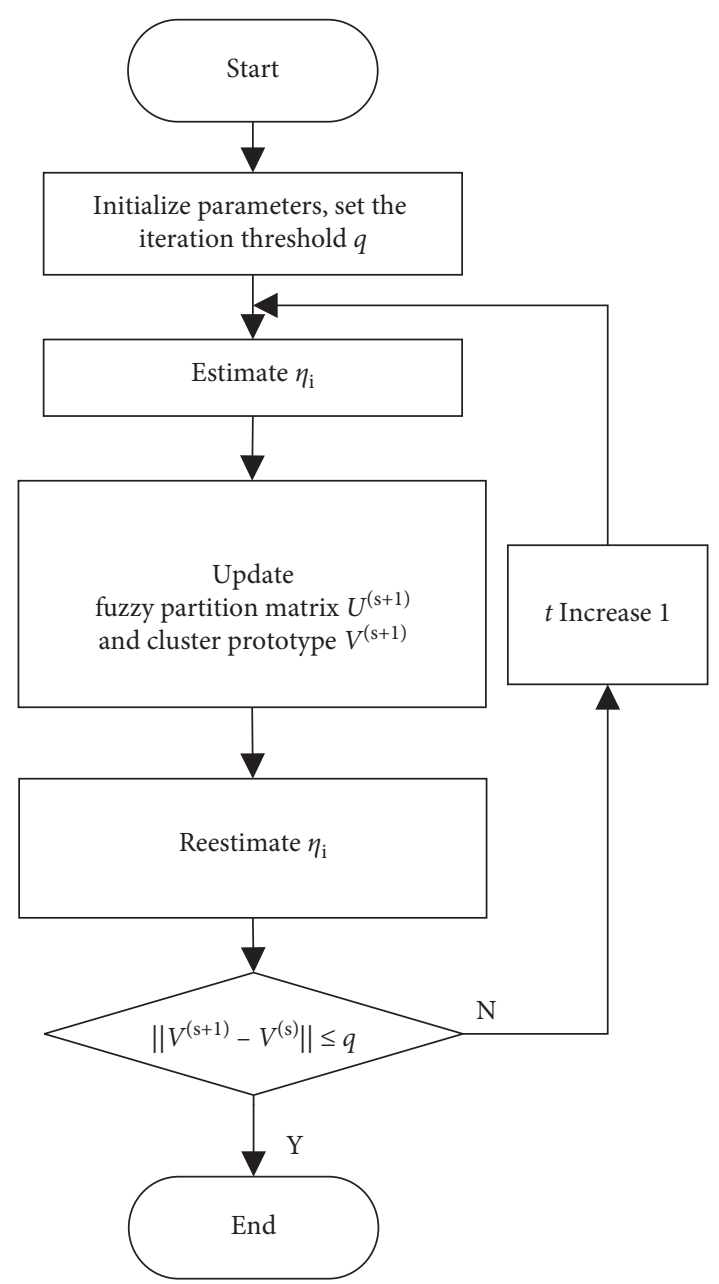

FIGURE 3: PCM algorithm calculation process.

the corresponding variance $\sigma_{i q}^{2}$ of $G_{i}$ can be expressed as $[7,8]$

$$
\begin{aligned}
v_{i q} & =\frac{\sum_{k=1}^{n}\left(u_{i k}\right)^{\beta} q_{k}}{\sum_{k=1}^{n}\left(u_{i k}\right)^{\beta}} \\
\sigma_{i q}^{2} & =\frac{\sum_{k=1}^{n}\left(u_{i k}\right)^{\beta}\left(q_{k}-v_{i q}\right)^{2}}{\sum_{k=1}^{n}\left(u_{i k}\right)^{\beta}}, \\
i & =1,2 \ldots c
\end{aligned}
$$

Considering the critical demand of the fuzzy clustering after dividing the input data space, the category data $q_{k}$ of $G_{i}$ and the corresponding variance $\sigma_{i q}^{2}$ of the category component $v_{i n+1}, i=1,2, \ldots c$ under the clustering center $V_{i}=$ $\left[v_{i 1}, v_{i 2} \ldots, v_{i n+1}\right]^{T}$ approximate 0 , thus using the minimal value (namely the shortest distance to the category center $v_{i q}$ ) of the category amount $q_{k}$ as the decision function $d_{F}\left(G_{i}\right)$ of the fuzzy clustering $d_{F}\left(G_{i}\right)$,

$$
d_{F}\left(G_{i}\right)=\left\{q_{k} \mid \min \left(\left|q_{k}-v_{i q}\right|\right)\right\}, \quad k=1,2 \ldots l, i=1,2 \ldots c .
$$


Correspondingly, the membership function $\mathrm{Gu}\left(x_{k}\right)$ of $G_{i}$ is expressed as

$$
\mathrm{Gu}_{j i}\left(x_{k j}\right)=\operatorname{Exp}\left(-\frac{\left|x_{k j}-v_{i q}\right|}{\left|v_{i q}-v_{i q}^{\prime}\right|} \times \gamma\right), \quad i=1,2, \ldots, c ; j=1,2, \ldots ., n .
$$

In the equation $\left|v_{i q}-v_{i q}^{\prime}\right|$ represents the width of the input data's division area, $v_{i q}$ represents the nearest cluster center value of cluster center No. $i$, factor $\gamma$ sets the rate of the membership decrease when the input samples fleeing the cluster center, normally $2 \leq \gamma \leq 4$.

The membership function $\mathrm{Gu}_{j i}\left(x_{k j}\right)$ represents the similarity between the input sample $x_{k}$ and a certain fuzzy class $G_{i}$. There is a relationship between the two: when $x_{k}$ is far away from the prototype, then $\mathrm{Gu}_{j i}\left(x_{k j}\right)$ is close to 0 ; when $x_{k}$ is close to the prototype, then $\mathrm{Gu}_{j i}\left(x_{k j}\right)$ is close to 1.

$R_{i}$ represents a decision rule of certain clustering category

$\left(R_{i}:\right.$ if $x_{k} \in G_{i}$, then $\left.\mathrm{d}\left(x_{k}\right)=\mathrm{d}_{F}\left(G_{i}\right), i=1,2 \ldots c\right)$, when the multidimensional fuzzy set $G_{i}$ projected onto the entire input data space, the decision rule $R_{i}^{\prime}$ can be expressed as

$$
\begin{gathered}
R_{i}^{\prime}: \text { If } x_{1} \in G_{i 1} \text { and } x_{2} \in G_{i 2} \text { and } \ldots \text { and } x_{n} \in G_{i n}, \\
\text { Then }\left(x_{k}\right)=\mathrm{d}_{F}\left(G_{i}\right), i=1,2 \ldots c
\end{gathered}
$$

The compatibility $\mathrm{G} \alpha_{i}$ of the corresponding $x_{k}$ for rule $R_{i}^{\prime}$ is the product of each component's membership $\mathrm{Gu}_{j i}\left(x_{k j}\right), j=1,2 . . n$ :

$$
G \alpha_{i}=\prod_{j=1}^{n} \mathrm{Gu}_{j i}\left(x_{k j}\right), i=1,2 \ldots c .
$$

As shown above, the membership function of the model is calculated by equation (9), the rule compatibility is calculated by equation (11).

$\sigma_{i q}^{2}$ manifests the quality of fuzzy division. The larger $\sigma_{i q}^{2}$ is, the worse quality the division is; thus, enter $E \sigma$ as the quality index of the fuzzy division. If $\sigma_{i q}^{2}>E \sigma, c=c+1$, a second fuzzy division is warranted. The relevant fuzzy clustering will not be extracted as the TS-FNN antecedent network until all the categories meet the condition $\sigma_{i q}^{2} \leq E \sigma$ [8].

After the building of the antecedent network is completed, estimates of the consequent network's linear parameter $p_{j i}^{r}$ are required. In this paper, the linear least squares recursive estimation method is used to estimate the parameters of the postnetwork. In this part of the training learning phase, the parameters of the antecedent network need to be fixed first.

Constructing error cost function is as

$$
E=\frac{1}{2}\left(y_{r}^{\prime}-y_{r}\right)^{2}
$$

where $y_{r}^{\prime}$ represents the expected output, $y_{r}$ represents the actual output.

Optimized estimate algorithm of $p_{j i}^{r}$.

$$
\begin{gathered}
\frac{\partial E}{\partial p_{j i}^{r}}=\frac{\partial E}{\partial y_{r}} \frac{\partial y_{r}}{\partial y_{g j}} \frac{\partial y_{g j}}{\partial p_{j i}^{r}}=-\overline{a_{j}}\left(y_{r}^{\prime}-y_{r}\right) x_{i}, \\
p_{j i}^{r}(j+1)=p_{j i}^{r}(j)-f \frac{\partial E}{\partial p_{j i}^{r}}=p_{j i}^{r}(j)+f \overline{a_{j}}\left(y_{r}^{\prime}-y_{r}\right) x_{i} .
\end{gathered}
$$

Among them, $a_{j}$ is the $j^{\text {th }}$ fuzzy rule adaptability, $\overline{a_{j}}=\left(a_{j} / \sum_{b=1}^{m} a_{b}\right) ; f \in(0,1), f$ is algorithm learning rate; $g=1,2, \ldots, r ; j=1,2, \ldots, m$.

\section{Model Verification Experiment and Test}

5.1. Construction of the Health Status Prognosis Model. In order to verify the efficiency of the health prognosis PTSFNN model for R2R processing device this paper proposed, the R2R continuous manufacturing device shown in Figure 4 is introduced as the experiment platform, its principle of operation is shown as Figure 5. Use the PCA parameter $x_{1}$, $x_{2}, x_{3}$, and $x_{4}$ of the roller performance corresponding to workstation 1, 2, 3, and 5 of the R2R manufacturing system as the input of the PTS-FNN model. Divide the thin film processing quality and the health status of the device in accordance with Table 1 and quantify to obtain the health status indicator function $y=\{1,2,3,4\}$ of the device as the output of PTS-FNN.

As shown in Figure 4, the R2R flexible material processing system adopted is mainly composed of a mechanical structure and drive control unit. For the mechanical structure, standard enforced aluminum alloy extrusions was selected as the supporting frame, including the unwinding module, the drive module, the wind-in module, etc. The experiment chose blue PET polyester film with thickness of $0.05 \mathrm{~mm}$, width of $50 \mathrm{~mm}$, elastic modulus of $3495 \mathrm{MPa}$, density of $1450 \mathrm{~kg} / \mathrm{m}^{3}$, and Poisson's ratio of 0.3 . The vibration data is collected by the AWA5936 vibration measuring instrument. The AWA5936 vibration measuring instrument has good repeatability and can ensure the accuracy of the measurement. The built-in acceleration sensor sensitivity is $1 \mathrm{pC} / \mathrm{m} / \mathrm{s}^{2} \pm 5 \%$, the measurement range of acceleration is $0.03 \mathrm{~m} / \mathrm{s}^{2}$ to $1000 \mathrm{~m} / \mathrm{s}^{2}$, the piezoelectric sensor frequency response range is $10 \mathrm{~Hz}$ to $8 \mathrm{kHz}$, the measurement error is $5 \%$ to $10 \%$ (LO : $10 \mathrm{~Hz}$ to $1 \mathrm{kH}$; HI: $1 \mathrm{kHz}$ to $8 \mathrm{kHz}$ ), and the sampling frequency is $48 \mathrm{kHz}$.

Principle of operation is shown as Figure 5. Based on different functions of each roll shaft, the R2R processing system is divided into different workstations. As mentioned in the previous content, the vibration workstation $1,2,3,4$, and 5 is measured, respectively, and the data is collected and documented every 30 minutes.

Roll shafts of four workstations used to evaluate the performance deterioration of the R2R device are of 4 different stages: excellent condition, prerecession, developing recession, and near expiry. Roll shaft of each stage demonstrates different vibration signals. The vibration signals of workstation1's unwinding roller at different performance stages is shown in Figure $6\left(\mathrm{~g}=9.8 \mathrm{~m} / \mathrm{s}^{2}\right)$. 

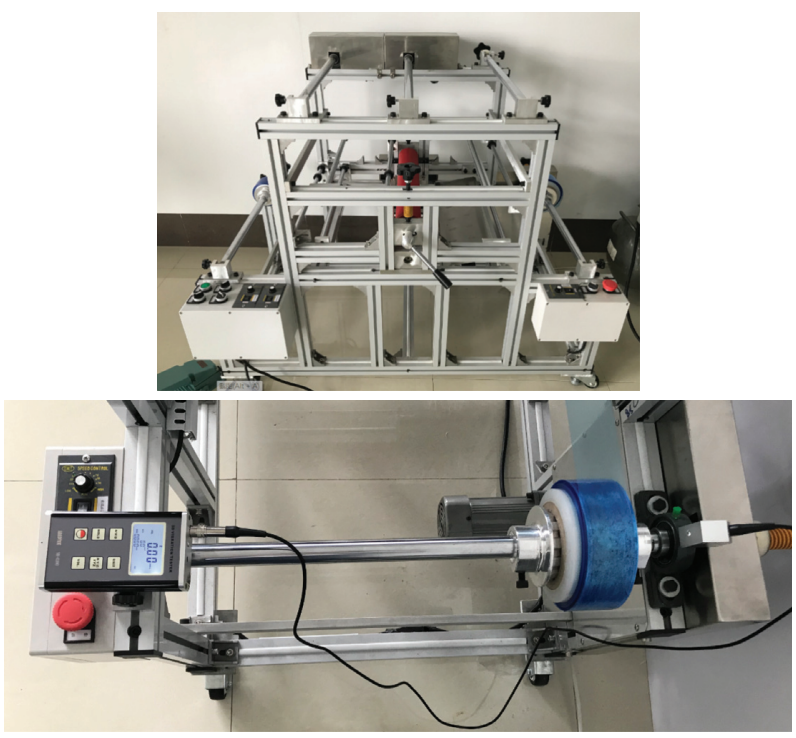

FIGURE 4: R2R continuous processing equipment.

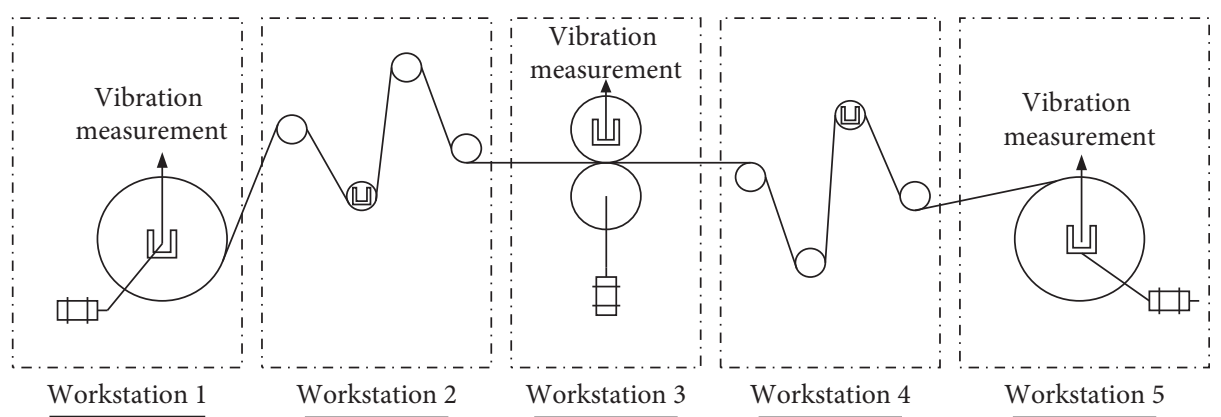

FIGURE 5: R2R system working principle diagram.

TABLE 1: Film processing quality and equipment health status.

\begin{tabular}{|c|c|c|c|}
\hline $\begin{array}{l}\text { Serial } \\
\text { number }\end{array}$ & Film processing quality & $\begin{array}{l}\text { Equipment health } \\
\text { status }\end{array}$ & $\begin{array}{c}\text { Device health status quantified } \\
\text { value }\end{array}$ \\
\hline 1 & $\begin{array}{l}\text { The number of pleats is } 0 \text { and the maximum offset of the winding } \\
\qquad \leq 1 \mathrm{~mm}\end{array}$ & Excellent condition & 1 \\
\hline 2 & $\begin{array}{l}\text { The number of pleats is } 1 \text { and the maximum offset of the winding } \\
\qquad \leq 1.5 \mathrm{~mm}\end{array}$ & Prerecession & 2 \\
\hline 3 & $\begin{array}{c}\text { The number of pleats is } 1-3 \text { and the maximum offset of the } \\
\text { winding } \leq 2 \mathrm{~mm}\end{array}$ & Developing recession & 3 \\
\hline 4 & More than 3 pleats or maximum offset $>2 \mathrm{~mm}$ & Near expiry & 4 \\
\hline
\end{tabular}

Collect 20 sets of data from each stage of each of the 4 workstations' roll shafts, 320 in total, as the experiment data of the performance deterioration prognosis PTSFNN model for the R2R processing device. Based on the building method mentioned in Section 3, make $c=2$, $E \sigma=1.25$, and start the fuzzy division of the input data. When $c=5$ and all variance of the fuzzy division categories meet the condition of $\sigma_{i q}^{2} \leq E \sigma$, the fuzzy division of the input data ends. Table 2 shows the 5 category centers after fuzzy division, which is expressed as $V_{i}, i=1,2, \ldots, 5$, and the according width of divides data regions $R_{i q}$ $\left(R_{i q}=\left|v_{i q}-v_{i q}^{\prime}\right|, v_{i q}\right.$ and $v_{i q}^{\prime}$ indicates the category component of $V_{i}, v_{i q}$ is the clustering center value nearest category center No. i).

After obtaining each of the category center and the width of the divided regions, according to equation (9), make $\gamma=2$, and the membership functions of input $(x)_{1} \sim(x)_{4}$ are shown in Table 3. 

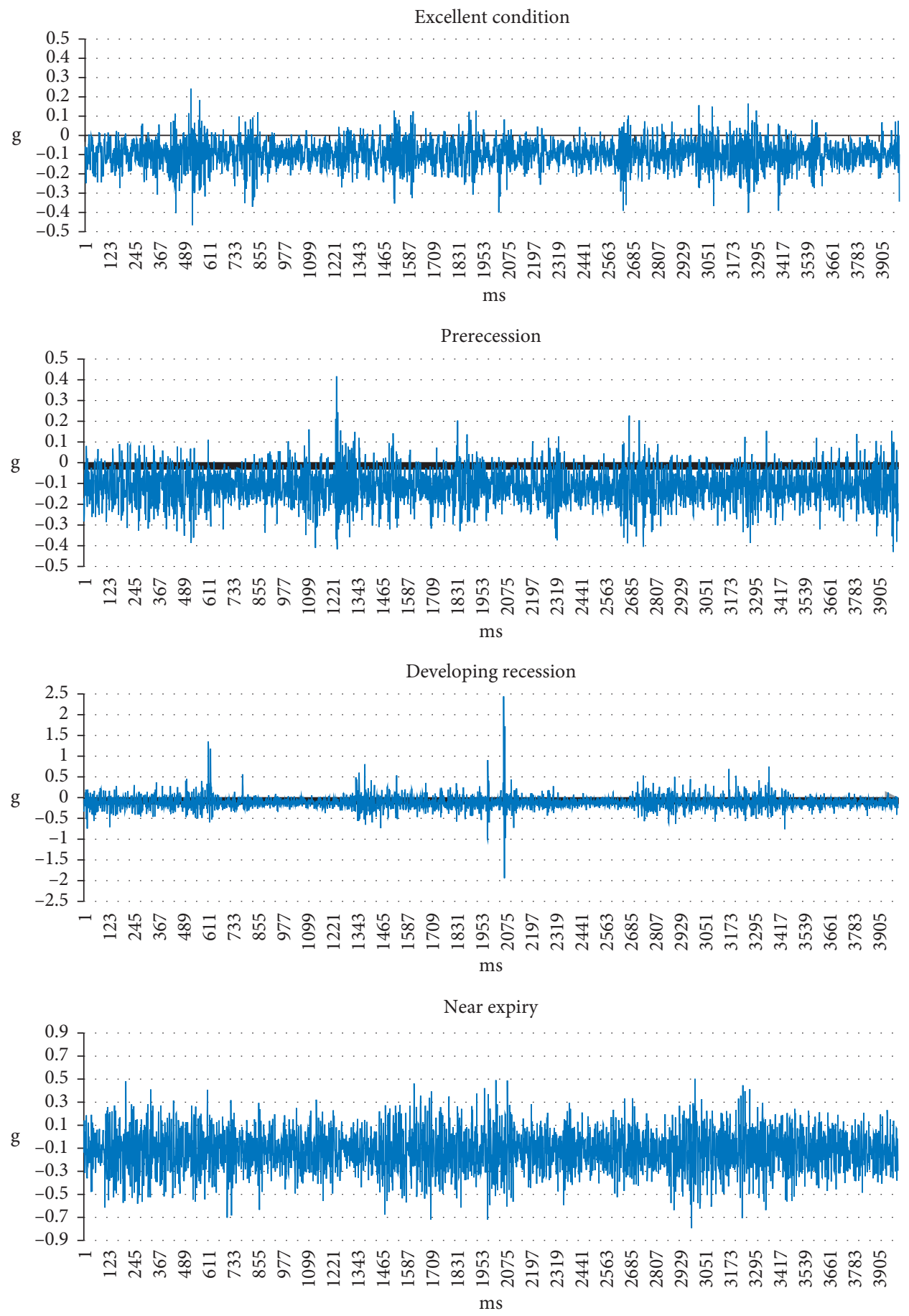

FIGURE 6: Vibration signal diagram of different performance conditions of the unwinding roller.

TABLE 2: Center coordinates of each category, the width of the division area.

\begin{tabular}{cccccccrc}
\hline & \multicolumn{3}{c}{ Center position coordinates } & \multicolumn{3}{c}{ The width of the division area } \\
$G_{i}$ & $v_{i x_{1}}$ & $v_{i x_{2}}$ & $v_{i x 3}$ & $v_{i x_{4}}$ & $R_{i x_{1}}$ & $R_{i x_{2}}$ & $R_{i x 3}$ \\
\hline$G_{1}$ & 2.0863 & 2.5407 & 0.0824 & 1.7858 & 1.0760 & 0.2052 & 0.1001 \\
$G_{2}$ & 1.4892 & 2.2923 & 0.0823 & 1.3848 & 1.0041 & 0.7030 & 0.0010 \\
$G_{3}$ & 2.8620 & 1.5765 & 0.0912 & 2.0093 & 0.7403 & 0.1750 & 0.5743 \\
$G_{4}$ & 1.8905 & 1.3779 & 0.0634 & 1.1342 & 1.5080 & 0.0003 & 0.1050 \\
$G_{5}$ & 2.7644 & 3.0037 & 0.0735 & 1.6871 & 1.5760 & 0.0510 & 0.1263 \\
\hline
\end{tabular}




$$
\begin{aligned}
& G \alpha_{1}=G u_{11}\left(x_{1}\right) \times G u_{21}\left(x_{2}\right) G u_{31}\left(x_{3}\right) G u_{41}\left(x_{4}\right)=\exp \left(-\frac{\left|x_{1}-2.0863\right|}{|1.0760|} \times 2\right) \times \exp \left(-\frac{\left|x_{2}-2.5407\right|}{|0.2052|} \times 2\right) \\
& \times \exp \left(-\frac{\left|x_{3}-0.0824\right|}{|0.1001|} \times 2\right) \times \exp \left(-\frac{\left|x_{4}-1.7858\right|}{|0.1263|} \times 2\right) \\
& G \alpha_{2}=G u_{12}\left(x_{1}\right) \times G u_{22}\left(x_{2}\right) G u_{32}\left(x_{3}\right) G u_{42}\left(x_{4}\right)=\exp \left(-\frac{\left|x_{1}-1.4892\right|}{|1.0041|} \times 2\right) \times \exp \left(-\frac{\left|x_{2}-2.2923\right|}{|0.7030|} \times 2\right) \\
& \times \exp \left(-\frac{\left|x_{3}-0.0823\right|}{|0.0010|} \times 2\right) \times \exp \left(-\frac{\left|x_{4}-1.3848\right|}{|0.1213|} \times 2\right) \\
& G \alpha_{3}=G u_{13}\left(x_{1}\right) \times G u_{23}\left(x_{2}\right) G u_{33}\left(x_{3}\right) G u_{43}\left(x_{4}\right)=\exp \left(-\frac{\left|x_{1}-2.8620\right|}{|0.7403|} \times 2\right) \times \exp \left(-\frac{\left|x_{2}-1.5765\right|}{|0.1750|} \times 2\right) \\
& \times \exp \left(-\frac{\left|x_{3}-0.0912\right|}{|0.5743|} \times 2\right) \times \exp \left(-\frac{\left|x_{4}-2.0093\right|}{|0.1261|} \times 2\right) \\
& G \alpha_{4}=G u_{14}\left(x_{1}\right) \times G u_{24}\left(x_{2}\right) G u_{34}\left(x_{3}\right) G u_{44}\left(x_{4}\right)=\exp \left(-\frac{\left|x_{1}-1.8905\right|}{|1.5080|} \times 2\right) \times \exp \left(-\frac{\left|x_{2}-1.3779\right|}{|0.0003|} \times 2\right) \\
& \times \exp \left(-\frac{\left|x_{3}-0.0634\right|}{|0.1050|} \times 2\right) \times \exp \left(-\frac{\left|x_{4}-1.1342\right|}{|0.1246|} \times 2\right) \\
& G \alpha_{5}=G u_{15}\left(x_{1}\right) \times G u_{25}\left(x_{2}\right) G u_{35}\left(x_{3}\right) G u_{45}\left(x_{4}\right)=\exp \left(-\frac{\left|x_{1}-2.7644\right|}{|1.5760|} \times 2\right) \times \exp \left(-\frac{\left|x_{2}-3.0037\right|}{|0.0510|} \times 2\right) \\
& \times \exp \left(-\frac{\left|x_{3}-0.0735\right|}{|0.3847|} \times 2\right) \times \exp \left(-\frac{\left|x_{4}-1.6871\right|}{|0.1175|} \times 2\right)
\end{aligned}
$$

Set neural nodes of the TS-FNN's antecedent network and consequent network for each layer referring to paper $[6,7]$; see Table 4 for details.

Select 240 sets of the 320 sample sets to train the consequent network, the remaining 80 sets of samples are used to inspect the accuracy of the model. Set the initial learning efficiency to 0.55 which decreases successively by 0.05 , the minimal expected learning error to 0.005 , the amount of training steps to 500. Then the linear parameter of the consequent network $p_{j i}^{r}$ is calculated (shown in Table 5).

5.2. Model Validation. In order to verify the validity of the PTS-FNN model proposed, select 240 sets of the 320 sample sets to build the PTS-FNN model and the standard TS-FNN [7] prognosis model, respectively, and repeat training both of the models 20 times each to establish the average time of model building and training. Training time of the former is 47.85 seconds while the latter is 96.43 seconds, which means the former model reduces the training time by $50.37 \%$ relatively.
The remaining 80 sets of samples are used to inspect the accuracy of the model. Table 6 shows a part of samples and testing data; the comparison of the accuracy of both models is shown in Table 7.

Comparing the two prediction models of standard TSFNN and PTS-FNN neural network, it can be concluded that in the training time, the latter is shorter than the training time of the former, which improves the convergence speed of the neural network and reduces the training time; In terms of accuracy, the prediction accuracy of the standard TS-FNN is $91.25 \%$, and the prediction accuracy of the PTS-FNN neural network is $96.25 \%$. The latter is more accurate than the former, and there is no error in the judgment of state 1 and state 4 .

\section{Conclusion}

Based on the analysis of the health prediction status of flexible photoelectric film processing equipment, this paper proposed a PTS-FNN for flexible material processing equipment health prediction combined with PCM and TSFNN. The model combined the advantages of PCM method, 
TABLe 3: Membership function of input quantity $(x)_{1} \sim(x)_{4}$. And according to equation (11) and Table 3, G $\alpha_{i}$ can be expressed as the equation given in the table.

\begin{tabular}{lccc}
\hline & $\begin{array}{c}\text { Input quantity } x_{1}, x_{2} \\
\text { Sembership function }\end{array}$ & $\begin{array}{c}\text { Input quantity } x_{3}, x_{4} \\
\text { Membership function }\end{array}$ \\
\hline 1 & $\mathrm{Gu}_{11}\left(x_{1}\right)=\exp \left(-\left(\left|x_{1}-2.0863\right| /|1.0760|\right) \times 2\right)$ & 11 & $\mathrm{Gu}_{31}\left(x_{3}\right)=\exp \left(-\left(\left|x_{3}-0.0824\right| /|0.1001|\right) \times 2\right)$ \\
2 & $\mathrm{Gu}_{12}\left(x_{1}\right)=\exp \left(-\left(\left|x_{1}-1.4892\right| /|1.0041|\right) \times 2\right)$ & 12 & $\mathrm{Gu}_{32}\left(x_{3}\right)=\exp \left(-\left(\left|x_{3}-0.0823\right| /|0.0010|\right) \times 2\right)$ \\
3 & $\mathrm{Gu}_{13}\left(x_{1}\right)=\exp \left(-\left(\left|x_{1}-2.8620\right| /|0.7403|\right) \times 2\right)$ & 13 & $\mathrm{Gu}_{33}\left(x_{3}\right)=\exp \left(-\left(\left|x_{3}-0.0912\right| /|0.5743|\right) \times 2\right)$ \\
4 & $\mathrm{Gu}_{14}\left(x_{1}\right)=\exp \left(-\left(\left|x_{1}-1.8905\right| /|1.5080|\right) \times 2\right)$ & 14 & $\mathrm{Gu}_{34}\left(x_{3}\right)=\exp \left(-\left(\left|x_{3}-0.0634\right| /|0.1050|\right) \times 2\right)$ \\
5 & $\mathrm{Gu}_{15}\left(x_{1}\right)=\exp \left(-\left(\left|x_{1}-2.7644\right| /|1.5760|\right) \times 2\right)$ & 15 & $\mathrm{Gu}_{35}\left(x_{3}\right)=\exp \left(-\left(\left|x_{3}-0.0735\right| /|0.3847|\right) \times 2\right)$ \\
6 & $\mathrm{Gu}_{21}\left(x_{2}\right)=\exp \left(-\left(\left|x_{2}-2.5407\right| /|0.2052|\right) \times 2\right)$ & 16 & $\mathrm{Gu}_{41}\left(x_{4}\right)=\exp \left(-\left(\left|x_{4}-1.7858\right| /|0.1263|\right) \times 2\right)$ \\
7 & $\mathrm{Gu}_{22}\left(x_{2}\right)=\exp \left(-\left(\left|x_{2}-2.2923\right| /|0.7030|\right) \times 2\right)$ & 17 & $\mathrm{Gu}_{42}\left(x_{4}\right)=\exp \left(-\left(\left|x_{4}-1.3848\right| /|0.1213|\right) \times 2\right)$ \\
8 & $\mathrm{Gu}_{23}\left(x_{2}\right)=\exp \left(-\left(\left|x_{2}-1.5765\right| /|0.1750|\right) \times 2\right)$ & 18 & $\mathrm{Gu}_{43}\left(x_{4}\right)=\exp \left(-\left(\left|x_{4}-2.0093\right| /|0.1261|\right) \times 2\right)$ \\
9 & $\mathrm{Gu}_{24}\left(x_{2}\right)=\exp \left(-\left(\left|x_{2}-1.3779\right| /|0.0003|\right) \times 2\right)$ & 19 & $\mathrm{Gu}_{44}\left(x_{4}\right)=\exp \left(-\left(\left|x_{4}-1.1342\right| /|0.1246|\right) \times 2\right)$ \\
10 & $\mathrm{Gu}_{25}\left(x_{2}\right)=\exp \left(-\left(\left|x_{2}-3.0037\right| /|0.0510|\right) \times 2\right)$ & 20 & $\mathrm{Gu}_{45}\left(x_{4}\right)=\exp \left(-\left(\left|x_{4}-1.6871\right| /|0.1175|\right) \times 2\right)$ \\
\hline
\end{tabular}

TABLE 4: Number of neurons in each layer of TS-FNN.

\begin{tabular}{lcccc}
\hline & First layer & Second layer & Third layer & Fourth layer \\
\hline Antecedent network & 4 & 20 & 5 & 5 \\
Consequent network & 5 & 11 & 5 & 1 \\
\hline
\end{tabular}

TABLE 5: Consequent network parameter training results.

\begin{tabular}{|c|c|c|c|c|c|c|c|c|c|}
\hline \multicolumn{5}{|c|}{$p_{1 i}^{1}$} & \multicolumn{5}{|c|}{$p_{2 i}^{2}$} \\
\hline 0.4773 & 0.0749 & 0.6731 & 0.0389 & 0.5961 & 0.3543 & 0.1951 & 0.3349 & -0.2759 & 0.6936 \\
\hline-0.0612 & 0.4454 & 0.3194 & 0.0080 & 0.6034 & -0.3855 & -0.0272 & -0.1542 & 0.2631 & -0.1080 \\
\hline 0.5493 & -0.0567 & -0.2279 & 0.2641 & -0.3716 & -0.0477 & 0.0297 & 0.5030 & 0.1217 & 0.3906 \\
\hline 0.6018 & 0.4309 & -0.1189 & 0.6352 & 0.2714 & 0.0866 & -0.2002 & 0.3345 & -0.2826 & 0.4592 \\
\hline 0.4378 & -0.1727 & 0.0229 & 0.4259 & -0.1466 & 0.1848 & 0.6407 & 0.2744 & -0.1019 & 0.3758 \\
\hline-0.2025 & 0.3485 & -0.1682 & 0.0041 & 0.0330 & 0.4103 & 0.5250 & 0.6120 & -0.2094 & 0.0872 \\
\hline-0.1528 & 0.0966 & 0.3042 & 0.4979 & -0.1313 & 0.5285 & 0.4929 & 0.0388 & 0.3712 & -0.0690 \\
\hline-0.0172 & 0.1219 & -0.1331 & 0.4291 & -0.2181 & 0.5231 & -0.2441 & 0.4129 & 0.4890 & 0.5448 \\
\hline-0.3210 & 0.6402 & 0.0462 & -0.0245 & -0.2632 & 0.4507 & -0.0911 & -0.2587 & 0.3511 & 0.4184 \\
\hline-0.1133 & -0.1793 & 0.2354 & 0.2057 & -0.0893 & 0.3479 & 0.5745 & 0.0471 & 0.4944 & 0.3721 \\
\hline 0.0780 & 0.4552 & 0.0436 & -0.1228 & -0.0188 & -0.3268 & 0.5609 & -0.0953 & -0.1683 & -0.3001 \\
\hline
\end{tabular}

TABle 6: Part of the sample and test data.

\begin{tabular}{|c|c|c|c|c|c|c|c|}
\hline Sample & $x_{1}$ & $x_{2}$ & $x_{3}$ & $x_{4}$ & PTS-FNN prediction result & TS-NN prediction result & Actual health status \\
\hline 1 & 0.0183 & 0.0181 & 0.0185 & 0.0181 & 1 & 1 & 1 \\
\hline 2 & 0.0186 & 0.0188 & 0.0188 & 0.0187 & 1 & 1 & 1 \\
\hline 3 & 0.0189 & 0.0187 & 0.1024 & 0.0943 & 3 & 2 & 3 \\
\hline 4 & 0.0321 & 0.0452 & 0.0187 & 0.0182 & 2 & 2 & 2 \\
\hline 5 & 0.0181 & 0.0190 & 0.0186 & 0.0182 & 1 & 1 & 1 \\
\hline 6 & 0.0186 & 0.0184 & 0.0188 & 0.0189 & 1 & 1 & 1 \\
\hline 7 & 0.0184 & 0.0816 & 0.0368 & 0.0988 & 3 & 2 & 3 \\
\hline 8 & 0.0357 & 0.0132 & 0.0189 & 0.0179 & 1 & 1 & 1 \\
\hline$\ldots$ & $\ldots$ & $\ldots$ & $\ldots$ & $\ldots$ & $\ldots$ & $\ldots$ & $\ldots$ \\
\hline 78 & 0.2240 & 0.4577 & 0.0183 & 0.2570 & 4 & 3 & 4 \\
\hline 79 & 0.0182 & 0.0187 & 0.0186 & 0.0189 & 1 & 1 & 1 \\
\hline 80 & 0.0377 & 0.0391 & 0.0450 & 0.2490 & 2 & 2 & 2 \\
\hline
\end{tabular}

TABle 7: Comparison of model prediction accuracy.

\begin{tabular}{cccccccccc}
\hline & & \multicolumn{3}{c}{ TS-FNN prediction result } & \multicolumn{3}{c}{ PTS-FNN prediction result } \\
& & State 1 & State 2 & State 3 & State 4 & State 1 & State 2 & State 3 & State 4 \\
\hline \multirow{3}{*}{ Actual health status } & State 1 & 42 & 1 & 0 & 0 & 43 & 0 & 0 \\
& State 2 & 0 & 14 & 2 & 0 & 0 & 15 & 1 & 0 \\
& State 3 & 0 & 3 & 11 & 0 & 0 & 2 & 12 & 0 \\
& State 4 & 0 & 0 & 1 & 6 & 0 & 0 & 0 \\
\hline
\end{tabular}


T-S fuzzy inference model, and fuzzy neural network modeling method. We also studied the PCM classification algorithm of input data of PTS-FNN model, the antecedent network of TS-FNN prediction model, and the construction method of postcomponent network. Finally, the implementation process of PCM classification algorithm and TSFNN prediction model was given.

The R2R processing equipment health prediction experiment system was built and the PTS-FNN model experiment was carried out. The experimental results showed that the training time of PTS-FNN model was $50.37 \%$ less than the standard TS-FNN prediction model. The prediction accuracy increased by $5.48 \%$, and the PTS-FNN had no in the judgment of state 1 and state 4 .

The proposed PTS-FNN health state prediction model was used for health state prediction of continuous manufacturing systems with time-varying and multistation features such as R2R processing equipment, which improved the real-time and accuracy of the prediction model. The main problem which plagued the large-scale manufacturing of flexible photovoltaic film materials was solved.

\section{Data Availability}

The data that support the findings of this study are available from the corresponding author on request.

\section{Conflicts of Interest}

The authors declare that they have no conflicts of interest.

\section{Authors' Contributions}

Yaohua Deng and Kexing Yao contributed equally to this work.

\section{Acknowledgments}

This work was supported in part by the National Natural Science Foundation of China under (Grant No. 51675109), Natural Science Foundation of Guangdong Province, China, under (Grant No. 2017A030313308), Provincial Science and Technology Plan Project of Guangdong Province, China, under (Grant No. 2016B010124002), Provincial Science and Technology Plan Project of Guangdong Province, China, under (Grant No. 2016B010112003), and Provincial Research and Development Project in Key Areas of Guangdong Province, China, under (Grant No. 2019B010154001).

\section{References}

[1] J. Lee, H. Davari, J. Singh, and V. Pandhare, "Industrial Artificial Intelligence for industry 4.0-based manufacturing systems," Manufacturing Letters, vol. 18, pp. 20-23, 2018.

[2] T. Xia, L. Xi, E. Pan, and J. Ni, "Reconfiguration-oriented opportunistic maintenance policy for reconfigurable manufacturing systems," Reliability Engineering \& System Safety, vol. 166, pp. 87-98, 2017.

[3] J. Lee, Di Yuan, and X. Jia, "Etc. Enhanced virtual metrology on chemical mechanical planarization process using an integrated model and data-driven approach," International Journal of Prognostics and Health Management, vol. 8, pp. 1-8, 2017.

[4] J. Ni and T. Xia, "etc. Operating load based real-time rolling Grey forecasting for machine health prognosis in dynamic maintenance schedule," Journal of Intelligent Manufacturing, vol. 26, pp. 269-280, 2015.

[5] Y. Deng, K. Yao, Q. Lu, and Q. Lu, "Research on prediction method of performance degradation of flexible optoelectronic film material processing equipment based on adaptive fuzzy clustering," Mathematical Problems in Engineering, vol. 2018, pp. 1-9, 2018.

[6] J. Zhao and Z. Ouyang, "Etc. Combined prediction on avionics state optimized by MAGA," Acta Armamentarii, vol. 37, no. 4, pp. 727-734, 2016.

[7] Y. Deng and G. Liu, "ATS-FNN modeling and simulation of FWP processing deformation compensation prediction," Journal of South China University of Technology (Natural Science Edition), vol. 40, no. 3, pp. 137-142, 2012.

[8] Y. Deng, Q. Lu, K. Yao, and N. Zhou, "Study on phosphor powder precipitation model in flexible material manufacturing process based on neuro-fuzzy networkflexible material manufacturing process based on neuro-fuzzy network," Optik, vol. 168, pp. 563-576, 2018.

[9] C. Lee, H. Lee, H. Seol, and Y. Park, "Evaluation of new service concepts using rough set theory and group analytic hierarchy process," Expert Systems with Applications, vol. 39, no. 3, pp. 3404-3412, 2012.

[10] R. Krishnapuram, "A possibilistic approach to clusterin," IEEE Transactions on Fuzzy Systems, vol. 1, no. 2, pp. 98-110, 1993.

[11] N. R. Pal, K. Pal, J. M. Keller, and J. C. Bezdek, "A possibilistic fuzzy c-means clustering algorithm," IEEE Transactions on Fuzzy Systems, vol. 13, no. 4, pp. 517-530, 2005.

[12] A. Bhagat, N. Kshirsagar, P. Khodke, K. Dongre, and S. Ali, "Penalty parameter selection for hierarchical data stream clustering," Procedia Computer Science, vol. 79, pp. 24-31, 2016. 